\section{Reply to the Comment of V. I. Stepanov "On the Osipov-Terentjev Calculation of the Viscosity of Liquid Crystals"}

\author{
M. A. Osipov* and E. M. Terentjev**
}

Z. Naturforsch. 47a, 627-628 (1992);

received December 19, 1991

In his comment on [1], V. J. Stepanov has presented a new expression for the flow-induced correction to the one-particle distribution function in nematic liquid crystals. With the help of this solution he obtained a simple expression for the rotational viscosity $\gamma_{1}$ which does not possess the exponential temperature dependence related to the overcoming a potential barrier, contrary to results of [1]. From the physical point of view this means that the rotating mesogenic molecule does not overcome the mean field potential barrier but prefers to rotate around the director.

We accept the mathematical arguments of the Comment and agree that the approximate solution of the stationary Fokker-Planck equation, proposed in [1] (see (2) of the Comment) appears to be incorrect close to singularity point due to the wrong assumption that the last term in the equation can be neglected.

At the same time one can not accept the statement of Stepanov that this is a revision of the main result of [1]. In fact, it was shown in [1] that the experimental observed exponential temperature variation of the rotational viscosity of nematics can not be determined by the overcoming of the mean-field potential barrier but is related to the activation-like temperature dependence of the "molecular friction" coefficient $\lambda$. This coefficient is the parameter of the mean-field theory, and it was calculated in [1] using the more general correlation function approach. This general conclu-

Reprint requests to M. A. Osipov, Institut für Theoretische Physik, Technische Universität Berlin, Hardenbergstr. 36, W-1000 Berlin 12.

* Institut für Theoretische Physik, Technische Universität Berlin, Hardenbergstr. 36, W-1000 Berlin 12.

Alexander von Humboldt Foundation Fellow, on leave from the Institute of Crystallography, Acad. Sci. USSR, Moscow 117333.

** Department of Physics, Case Western Reserve University, Cleveland, Ohio 44106-7079, USA.

On leave from the Institute of Crystallography, Acad. Sci. USSR. sion is supported by the experiment since both the absolute value and the temperature variation of the viscosity activation energy are similar to those of the "molecular friction" coefficient, while the value and temperature variation of the mean field potential barrier are different. This result is not affected by the critisizm.

The Comment of Stepanov is related only to the temperature variation of the ratio of two viscosity coefficients $\gamma_{1} / \gamma_{2}$. According to [1] the ratio should also possess the exponential temperature dependence with an activation energy proportional to the mean field potential barrier $U_{0}$. This result has to be revised now.

It should be noted that, according to the Comment, the rotational viscosity $\gamma_{1} \approx c \lambda$ in the limit of large $q$, where $q=U_{0} / k T$ is the dimensionless mean-field potential barrier. At the same time the viscosity coefficient $\gamma_{2}$ is a growing function of $S[2,3]$. Taking $\gamma_{2}=c \lambda S$, as calculated by Kuzui and Doi [3], we arrive at the very simple relation $-\gamma_{1} / \gamma_{2} \sim S^{-1}$, where $S$ is the nematic order parameter. Consequently, according to the Comment, the ratio of two rotational viscosity coefficients should decrease with increasing temperature in the nematic phase. However, the experimental behaviour is the opposite, i.e., the ratio increases with decreasing temperature (see, e.g. Fig. 11 of [1]).

Thus we arrive at a strong contradiction between the theory and experiment. However, this contradiction can be explained satisfactorily if one assumes that the limit of large $q$ (i.e. the limit of very high potential barrier), which has been used both in [1] and in the Comment, is inadequate for real nematics. Indeed, the mean field potential barrier can be estimated using the Maier-Saupe theory which yields $q=U_{0} / k T \approx$ $J_{0} S / k T$ with $J_{0} \approx 4.5 k T_{\mathrm{c}}$. Then $q \approx 3$ for $S=0.7$, i.e. $q$ can probably be not considered as a large parameter even far from the transition point. In real systems the potential barrier can be even smaller [4]. Then it is reasonable to use another limit, i.e. to expand the coefficients $\gamma_{1}$ and $\gamma_{2}$ in powers of $S$. This expansion has been originally performed by Hess [2], and in lower order in $S$, one has $\gamma_{1} \sim S^{2} \tau, \gamma_{2} \sim S \tau_{1}$. Therefore, the ratio of two viscosity coefficients is now proportional to the nematic order parameter $S,-\gamma_{1} / \gamma_{2} \sim S$. One can readily see from Fig. 14 of [4] that this result is qualitative agreement with the experiment. 
In conclusion it should be noted that the general physical consequence of the Comment is very interesting and it strongly affects the present understanding of the molecular rotational dynamics in nematics. For example, the popular theory of nematics viscosity, proposed by Diogo and Martins [5, 6], is based on the same assumption that the molecules overcome the mean-field potential barrier. A similar assumption is

[1] M. A. Osipov and E. M. Terentjev, Z. Naturforsch. 44a, 785 (1989).

[2] S. Hess, Z. Naturforsch. 31 a, 1034 (1976).

[3] N. Kuzui and M. Doi, J. Phys. Soc. Japan 52, 3486 (1983). used also in the interpretation of the experimental data on relaxation times.

\section{Acknowledgements}

The authors are gratefull to V. I. Stepanov for his valuable Comment and to Prof. S. Hess for interesting discussions.

[4] S.-T. Wu and C.-S. Wu, Phys. Rev. A 42, 2219 (1990).

[5] A. Martins and A. Diogo, Portugal Phys. 9, 1 (1975).

[6] A. Diogo and A. Martins, Mol. Cryst. Liq. Cryst. 66, 113 (1981). 